\title{
Reconnaissance sous-marine de l'évolution des fonds en faible profondeur
}

Jean-Marc Rousset

Doctorant, Laboratoire de Mécanique, Université de Caen

Eric Vasselin

Maître de Conférences, Laboratoire d'Automatique de Commande des Systèmes, Université du Havre

Serge Simon

Cellule de Suivi du Littoral Haut-Normand, Le Havre

Résumé

Ce présent papier traite des possibilités offertes par l'utilisation de plongeurs scientifiques dans des études physiques du milieu marin, notamment la souplesse de mise en cuvre des moyens et la qualité des observations. Un exemple est présenté concernant la reconnaissance de l'évolution des fonds d'une gravière expérimentale au large du Havre.

\section{Introduction}

\subsection{Justification de telles études}

L'évolution des fonds marins en zone côtière est une donnée économique importante. Les nombreuses études qui s'y rapportent font souvent l'objet d'une démarche pluridisciplinaire. Les différentes composantes scientifiques collaborent et les observations in-situ complètent alors les modélisations physiques ou numériques des variations des fonds.

\subsection{Méthodes disponibles}

La plupart des campagnes de mesures impliquent l'utilisation de matériels de prélèvement (bennes), de topographie (sondeur, sonar latéral) et de courantométric. Cet appareillage nécessite donc l'armement d'un navire assez important représentant ainsi un coût non négligeable.

Pour le suivi régulier d'une zone d'étude, une alternative à cette approche "lourde" peut être constituée par des interventions sous-marines de plongeurs à partir d'un bateau de très faible tonnage.

Les biologistes marins utilisent cette technique avec satisfaction depuis de nombreuses années. Dans le cas d'études techniques, il est intéressant d'avoir recours à des plongeurs scientifiques eux-mêmes mécaniciens des fluides ou instrumentalistes de formation. En effet leurs sensibilités différentes de celle des biologistes induisent une approche novatrice des observations. 
Une fois sur le site concerné, les plongeurs sont à même de déterminer la topographie et la nature des fonds, de procéder à des prélèvements ponctuels ou d'installer de l'instrumentation légère (houlographe, marégraphe,...).

L'utilisation de moyens audiovisuels donne en outre une vision nette de la zone étudiée et autorise une exploitation instantanée des enregistrements sur le bateau même. Les gains de temps et de précision accroissent ainsi la qualité de l'étude.

\section{Reconnaissance de la "souille CNEXO"}

Un exemple d'une telle démarche est constitué par la mission sur la "souille

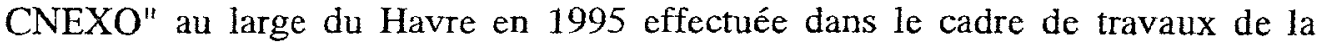
Commission interrégionale de concertation pour la gestion de la Baie de Seine.

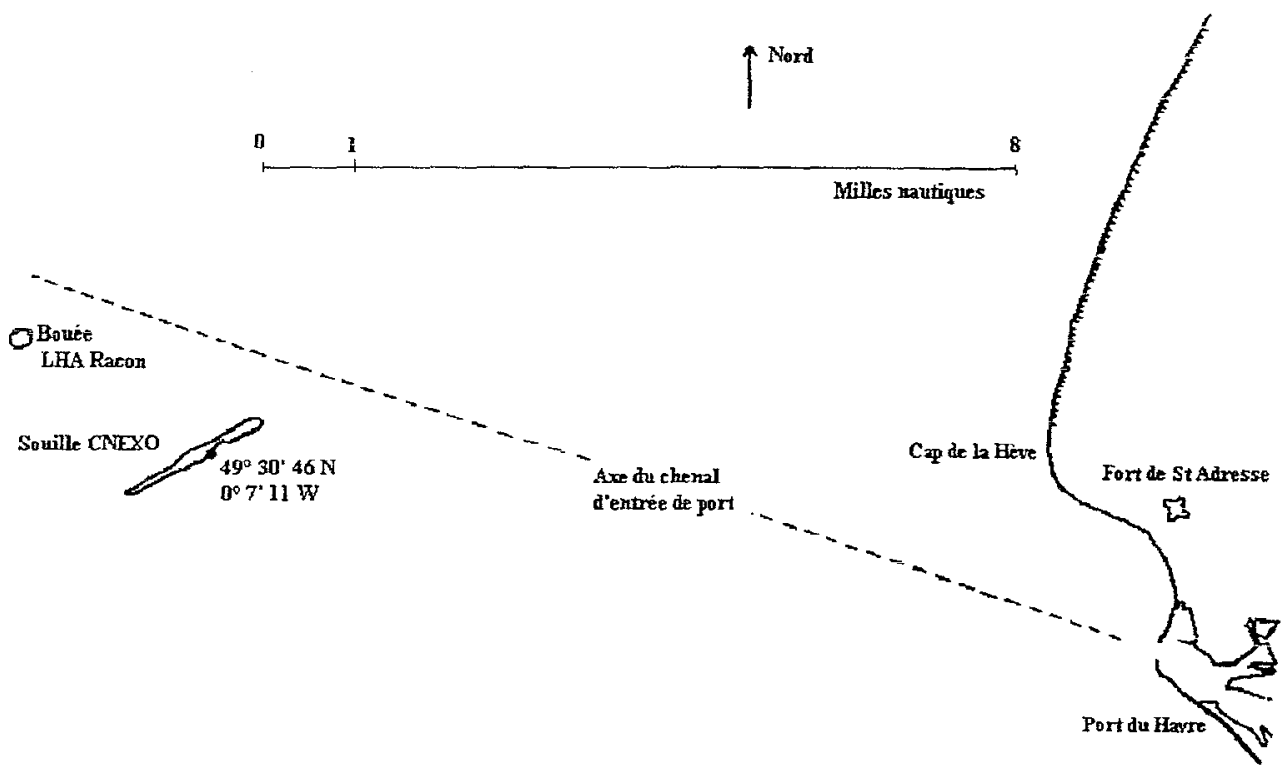

Figure 1: Situation de la "souille CNEXO" par rapport au chenal du port du Havre

\subsection{Historique de la zone}

Devant l'augmentation constante de la consommation annuelle française de sables et graviers, il avait été envisagé d'extraire les agrégats par dragage du plateau continental. La remise en suspension des fines particules $(30-40 \mu \mathrm{m})$ lors du travail de la drague impliquait certainement des répercussions sur l'environnement marin.

Le Centre National pour l'EXploitation des Océans (devenu depuis IFREMER) avait donc décidé d'appréhender expérimentalement ce problème. Afin d'altérer le milieu marin et d'en quantifier les conséquences, une zone fut déterminée sur la 
base de ses réserves en agrégats et de son environnement biologique diversifié. Localisé à 9 milles nautiques au nord-ouest du Havre (figure 1), le site a été utilisé dans les conditions d'une exploitation industrielle entre 1974 et 1980 en collaboration avec l'entreprise Gagneraud (Société des Graves de l'Estuaire).

\subsection{Description de la mission}

\subsubsection{Objectif}

En Mars 1995, la Cellule de Suivi du Littoral Haut-Normand a été mandatée par le Bureau des Recherches Géologiques et Minières pour effectuer un examen des fonds de cette souille expérimentale afin de rendre compte de son état biologique et de son état sédimentaire depuis l'arrêt de son exploitation. Cette mission consistait essentiellement en une reconnaissance vidéo de la zone.

Deux campagnes à la mer ont été faites entre Avril et Juin 1995, les conditions météorologiques défavorables obligeant à des reports successifs.

\subsubsection{Moyens humains}

Les plongées et les prises de vues ont été réalisées par des personnels du Laboratoire d'Etudes et d'Analyses de la Ville du Havre (LEA) et du Laboratoire de Mécanique des Fluides et Génie Civil de I'Université du Havre (LMFGC, intégré depuis au Laboratoire de Mécanique de l'Université de Caen).

\subsubsection{Moyens techniques}

\subsubsection{Navire}

L'embarcation est une barge de travail non pontée appartenant au LEA. Le positionnement est réalisé grâce aux systèmes GPS et TORAN. Le sondeur de bord permet de déterminer les contours de la souille et de définir les zones à reconnaître.

\subsubsection{Plongée}

La profondeur étant relativement faible, le matériel de plongée est constitué de l'équiipement classique de plongée autonome à l'air comprimé.

\subsubsection{Vidéo}

Deux types de caméras vidéos ont été utilisées durant les campagnes.

La première est une caméra filaire dont les images sont instantanément projetées sur un moniteur vidéo et enregistrées sur un magnétoscope VHS en surface. Un éclairage de 50 Watts est incorporé au bâti de la caméra ainsi qu'une liaison phonique unidirectionnelle surface-plongeur. Ces différentes liaisons filaires sont regroupées dans un câble d'une longueur de 100 mètres, se déroulant à partir du navire. Un petit groupe électrogène assure l'alimentation de l'ensemble. Cette installation reste compacte et embarquable sur une petite embarcation. Il est seulement nécessaire de la protéger des embruns. 
La deuxième est une caméra autonome constituée d'un camescope Hi-8mm protégée par un caisson aluminium Amphibian étanche jusqu'à 100 mètres. Deux spots halogènes de 50 Watts chacun, alimentés par une batterie placée dans le dos du plongeur, permettent d'obtenir une luminosité suffisante sur un large champ de vision.

\subsection{Méthodologie}

\subsubsection{En surface}

Le moniteur vidéo de contrôle permet aux intervenants non plongeurs (scientifiques, entrepreneurs,...), embarqués lors de la mission, d'étudier en direct ou en léger différé (quelques dizaines de minutes) les images de la zone. Les commentaires et les observations des plongeurs sont alors une aide importante pour la compréhension des observations. Il peut alors être décidé d'approfondir l'examen de celle-ci ou au contraire de reconnaître une autre zone par un autre plongée.

\subsubsection{Au fond}

Afin de matérialiser les zones que les plongeurs doivent inspecter, deux corpsmorts sont implantés en des points de coordonnées établies par GPS et TORAN. Ils sont signalés en surface par des bouées et reliés par une ligne plombée reposant sur le fond. Les sites de prospection correspondent à l'axe principal de la souille et à un axe transversal (figure 2). Ils sont compris entre 20 et 35 mètres de profondeur.

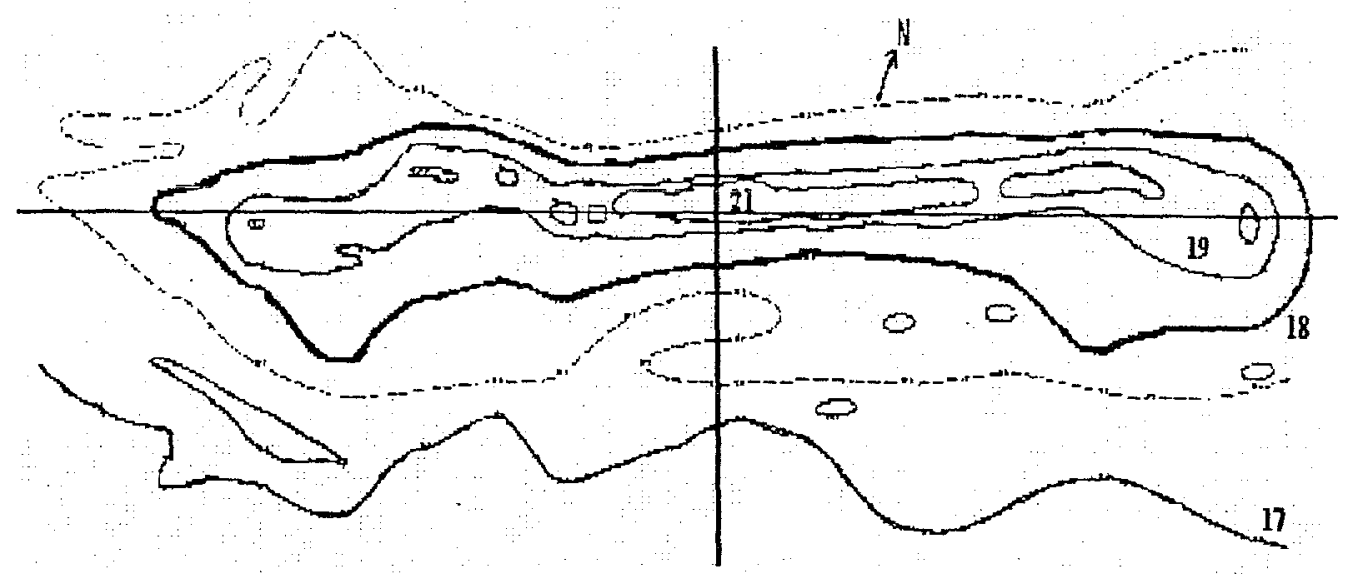

Figure 2 : Schéma de la bathymétrie à l'arrêt de l'exploitation et axes des progressions sous-marines. 
Les prélèvements biologiques et sédimentaires sont effectués ponctuellement par un deuxième plongeur aux endroits supposés les plus caractéristiques, au cours de la progression sur le fond. L'épaisseur de la couche vaseuse est évaluée par la pénétration d'une mire graduée de quelques dizaines de centimètres. Ces opérations sont filmées, les intervenants scientifiques non-plongeurs pouvant ainsi examiner les zones de prélèvements sur le moniteur vidéo.

\subsection{Résultats}

\subsubsection{Aspects sédimentaire et rocheux}

L'envasement $d u$ fond est homogène et recouvre entièrement les zones d'extraction des sables et graviers. Le dépôt est constitué d'argile compacte dont l'épaisseur varie entre 5-10 centimètres et 20-25 centimètres dans les dépressions. Les forts courants de marée (mesures de 1975 et observation des plongeurs) peuvent en partie expliquer la relative faiblesse de ce dépôt.

Les bords de la souille sont encore marqués par une forte déclivité et par un dépôt de sédiment de faible épaisseur.

Le centre de la souille ne renferme que très peu de gros blocs. Les plus importants mesurent moins d'un mètre dans leur plus grande dimension mais en moyenne ils sont d'un diamètre d'une dizaine de centimètres.

\subsubsection{Aspect biologique}

Les nombreux trous de vers fouisseurs et de bivalves observés dans la couche sédimentaire témoignent d'une grande variété d'invertébrés. Les blocs sont tous colonisés par des anémones de mer (Anthozoaires), des alcyones des éponges et des Hydrozoaires. De très nombreux Crustacés (étrilles, tourteaux, araignées, pagures) peuplent le fond de la souille dans les zones avec ou sans blocs. Les observations démontrent de plus la présence de Mollusques (seiches avec leurs pontes), de Poissons d'espèces commerciales (églefin, limande) et d'espèces communes (tacauds, labres, dragonnets...).

\subsubsection{Conclusion}

Au terme de la reconnaissance vidéo de la "souille CNEXO", il apparaît que le comblement de celle-ci est particulièrement lent, conformément aux hypothèses formulées en 1974-1975 ne le prévoyant pas avant 55 ans dans les conditions les plus favorables.

Il est établi par ailleurs que la faune sous-marine a repris possession de la zone prospectée, même si nous ne disposons de l'état biologique initial.

Ces observations qualitatives plus que quantitatives devraient cependant aider à la concertation entre les différents intervenants (industriels, pêcheurs, collectivités locales) quant à la gestion des graves en mer. 


\section{Conclusion}

L'approche physique, couplée aux aspects biologiques, donne une vision plus globale des problèmes environnementaux et dynamise les relations interdisciplinaires.

La reconnaissance sous-marine de l'évolution des fonds en faible profondeur implique des moyens relativement simples à mettre en cuvre, pour des résultats satisfaisants.

Une telle méthode ne remplace pas une étude approfondie et quantitative des conditions sédimentologiques et océanologiques d'un site, mais au contraire elle la complète.

Aux vues des possibilités offertes par cette approche, le programme européen de recherche MAST III Proverbs a encouragé un suivi de l'état des bermes de digues verticales, en France et à l'étranger, par des plongeurs scientifiques.

\section{Références}

CNEXO(1975): Etudes des gravières marines. Rapport d'étude(5 fascicules).

CSLHN (1995): Reconnaissance vidéo de la souille CNEXO. Rapport au BRGM du $7 / 07 / 95$. 